\title{
THE NUMBER OF VALUES OF COMBINATORIAL FUNCTIONS
}

\author{
RUDOLF AHLSWEDE AND DAVID E. DAYKIN
}

\section{Introduction}

An important discovery of Marica-Schönheim [5] is

THEOREM 1. Any $m$ distinct sets have at least $m$ distinct differences.

This result has various generalisations $[1-4]$ and one of a new kind is

THEOREM 2. If $S_{1}, \ldots, S_{m}$ are distinct sets, and $T_{1}, \ldots, T_{n}$ are sets such that each $S_{i}$ has some $T_{j}$ as a subset, then there are at least $m$ distinct differences $S_{i} \backslash T_{j}$.

The form of this theorem led us to conjecture that $m$ sets can be partitioned into $\mathfrak{S}$ and $\mathfrak{I}$ having $m-1$ differences $S \backslash T$ with $S \in \mathfrak{S}, T \in \mathfrak{I}$. Given an ordered sequence $S_{1}, \ldots, S_{m}$ of sets let $d$ be the number of differences $S_{i} \backslash S_{j}$ with $i<j$ and $e$ be the largest $n$ for which there are $S_{i_{1}} \subset \ldots \subset S_{i_{n}}$ with $1 \leqslant i_{1}<\ldots<i_{n} \leqslant m$. We believe $m, d, e$ are related.

The generalisation of Theorem 1 by Daykin-Lovász [4] is

THEOREM 3. Any non-trivial Boolean function takes at least $m$ distinct values when evaluated over $m$ distinct sets.

We give a generalisation of this theorem, which also yields a new proof.

\section{Proof of Theorem 2}

We may assume all the sets $S_{i}, T_{j}$ are subsets of $\{1,2, \ldots, r\}$ and use induction on $r$. The case $r=1$ is trivial. Put $\mathfrak{S}=\left\{S_{1}, \ldots, S_{m}\right\}, \mathfrak{I}=\left\{T_{1}, \ldots, T_{n}\right\}, \mathfrak{U}=\{S \backslash r: S \backslash r \in \mathfrak{S}$ and $S \cup r \in \mathfrak{S}\}, \mathfrak{B}=\{S \backslash r: S \in \mathfrak{S}\}, \mathbb{C}=\{T: r \notin T \in \mathfrak{I}\}$ and $\mathfrak{D}=\{T \backslash r: T \in \mathfrak{I}\}$. Then $m=|\mathfrak{U}|+|\mathfrak{B}|$, where $|$.$| denotes cardinality. Also \mathfrak{U}, \mathfrak{C}$ and $\mathfrak{B}, \mathfrak{D}$ satisfy the hypothesis on $\{1,2, \ldots, r-1\}$ so $|\mathfrak{U}| \leqslant|\mathfrak{U} \backslash \mathfrak{C}|$ and $|\mathfrak{B}| \leqslant|\mathfrak{B} \backslash \mathfrak{D}|$. If $E \in \mathfrak{U} \backslash \mathfrak{C}$ then $E=A \backslash C$ for some $A \in \mathfrak{U}, C \in \mathbb{C}$. Thus $A \backslash r, A \cup r \in \mathfrak{S}$ and $r \notin C \in \mathfrak{I}$ so $E \backslash r$, $E \cup r \in \mathfrak{S} \backslash \mathfrak{I}$. On the other hand if $E \in \mathfrak{B} \backslash \mathfrak{D}$ then clearly either $E \backslash r$ or $E \cup r$ is in $\mathfrak{S} \backslash \mathfrak{I}$. Hence $|\mathfrak{U} \backslash \mathfrak{C}|+|\mathfrak{B} \backslash \mathfrak{D}| \leqslant|\mathfrak{S} \backslash \mathfrak{I}|$ and the result follows.

\section{Generalisation of Theorem 3}

Let $c$ be a fixed positive integer. If $S$ is a set then $S^{c}$ denotes the set of all $c$-dimensional vectors with elements in $S$, and a $c$-ary operation $f$ on $S$ is a mapping $f: S^{c} \rightarrow S$. Given such a map $f$ for $A_{1}, \ldots, A_{c} \subset S$ put

$$
f\left(A_{1}, \ldots, A_{c}\right)=\left\{f\left(a_{1}, \ldots, a_{c}\right): a_{i} \in A_{i} \quad \text { for } \quad 1 \leqslant i \leqslant c\right\} .
$$

Received 3 January 1978.

[Bull. London Math. Soc., 11 (1979), 49-51] 
Call $f$ expansive if

$$
|A| \leqslant|f(A, \ldots, A)| \quad \text { for all } \quad A \subset S .
$$

Call $f$ c-expansive if

$$
\left|A_{1}\right| \leqslant\left|f\left(A_{1}, \ldots, A_{c}\right)\right| \text { for all } A_{1}, \ldots, A_{c} \subset S \text { with }\left|A_{1}\right|=\ldots=\left|A_{c}\right| .
$$

Notice that when $|S|=2$ expansive is the same as $c$-expansive and simply means non-constant Boolean function.

If $S, T$ are sets and $f: S^{c} \rightarrow S$ while $g: T^{c} \rightarrow T$ we define the direct product $h$ of $f$ and $g$ to be the map $h:(S \times T)^{c} \rightarrow S \times T$ such that

$$
h\left(\left(s_{1}, t_{1}\right), \ldots,\left(s_{c}, t_{c}\right)\right)=\left(f\left(s_{1}, \ldots, s_{c}\right), g\left(t_{1}, \ldots, t_{c}\right)\right) \text { for all } s_{i} \in S \text { and } t_{j} \in T \text {. }
$$

The direct product of expansive maps is not expansive, for example let $c=2, S=\{0$, $1,2\}, f(a, b)=\max \{0, a-b\}$, take the direct product of $f$ with itself and $A=(S \times S) \backslash\{(0,0),(2,2)\}$. It would be interesting to have more results like

THEOREM 4. In the above notation, if $f$ is expansive and $g$ is c-expansive then $h$ is expansive.

Proof. If $B \subset S \times T$ and $m$ is a positive integer let $B_{m}$ be the set of all $s \in S$ such that $(s, t) \in B$ for at least $m$ different $t \in T$. Let $A \subset S \times T$ be given and $x \in f\left(A_{m}, \ldots, A_{m}\right)$. Thus there are $s_{1}, \ldots, s_{c} \in A_{m}$ with $x=f\left(s_{1}, \ldots, s_{c}\right)$. For $1 \leqslant i \leqslant c$ there are distinct $t_{i 1}, \ldots, t_{i m} \in T$ with $\left(s_{i}, t_{i j}\right) \in A$ for $1 \leqslant j \leqslant m$. By hypothesis on $g$ we have

$$
m \leqslant\left|\left\{g\left(t_{1 j_{1}}, \ldots, t_{c j_{0}}\right): 1 \leqslant j_{1}, \ldots, j_{c} \leqslant m\right\}\right|
$$

and this means that $x \in(h(A, \ldots, A))_{m}$. Finally

$$
|A|=\sum\left|A_{m}\right| \leqslant \sum\left|f\left(A_{m}, \ldots, A_{m}\right)\right| \leqslant \sum\left|(h(A, \ldots, A))_{m}\right|=|h(A, \ldots, A)|
$$

and the proof is complete.

Now let $|S|=2$ and $f_{1}, \ldots, f_{n}: S^{c} \rightarrow S$. Further let $\mathfrak{N}, \mathfrak{P}$ be the set of all matrices of order $n \times c, n \times 1$ respectively with elements in $S$. Define $e: \mathfrak{N} \rightarrow \mathfrak{P}$ by

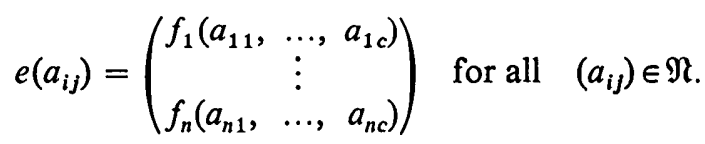

By induction on $n$ we immediately get from Theorem 4 that if $f_{1}, \ldots, f_{n}$ are non-constant then $e$ is expansive. The case of this with $f_{1}=\ldots=f_{n}$ is Theorem 3 .

\section{References}

1. R. Ahlswede and D. E. Daykin, "An inequality for the weights of two families of sets, their unions and intersections ", Z. Wahrecheinlichkeitstheorie und Verw. Gebiete, 43 (1978), 183-185.

2. D. E. Daykin, "A lattice is distributive iff $|A||B| \leqslant|A \vee B||A \wedge B|$ ", Nanta Math., 10 (1977), 58-60.

3. D. E. Daykin, "Functions on a distributive lattice with a polarity", J. London Math. Soc. (to appear). 
4. D. E. Daykin and L. Lovász, "The number of values of a Boolean function ", J. London Math. Soc., 12 (1976), 225-230.

5. J. Marica and J. Schönheim, "Differences of sets and a problem of Graham ", Canad. Math. Bull., 12 (1969), 635-637.

Fakultät für Mathematik,

Universität Bielefeld 4800,

F.D.R.

Department of Mathematics,

University of Reading,

G.B. 